\title{
Lebensqualität bei limitiertem Prostatakarzinom
}

Wie sich verschiedene Therapiestrategien beim lokal begrenzten Prostatakarzinom auf die Lebensqualität auswirken, wurde in der ProtecT-Studie geprüft.

$\mathrm{n}$ der ProtecT-Studie wurden $1.643 \mathrm{~Pa}-$ tienten mit Instrumenten wie ICSmaleSF, SF-12, HADS, ICIQ und EORTCQLQ-C30 befragt. Sie waren wegen eines lokal begrenzten Prostatakarzinoms auf eine von drei Strategien randomisiert worden: aktives Monitoring (regelmäßiger PSA-Test und klinische Untersuchung mit der Option, auf eine radikale Therapie $\mathrm{zu}$ wechseln), radikale Prostatektomie (meist offen retropubisch, nervenschonend) und Radiotherapie (externe 3-D-Bestrahlung mit 74 Gy in 37 Fraktionen zusammen mit neoadjuvanter Androgendeprivation).

Während des sechsjährigen Followups wirkte sich die Prostatektomie von allen drei Strategien am negativsten auf Sexualfunktion und Harnkontinenz aus. Auch wenn sich ein Teil der Patienten im Laufe der Zeit davon erholte, blieben die Funktionen doch über die gesamte Nach- beobachtungszeit schlechter als in den anderen Gruppen. Negative Auswirkungen der Radiotherapie auf die Sexualfunktion waren nach sechs Monaten am stärksten ausgeprägt, anschließend trat eine Erholung und Stabilisierung ein. Auf Harnkontinenz hatte die Radiotherapie nur geringe Auswirkungen. Dagegen waren Harnverhalt und Nykturie nach sechs Monaten in der Radiotherapiegruppe am stärksten ausgeprägt, die anschließende Erholung führte nach zwölf Monaten zu ähnlichen Werten wie in den anderen Gruppen. In der Beobachtungsgruppe verschlechterten sich Sexualund Harnfunktionen nur graduell.

Die Darmfunktion war nach sechs Monaten in der Radiotherapiegruppe schlechter als in den beiden anderen Gruppen. Sie verbesserte sich anschließend, mit Ausnahme einer erhöhten Häufigkeit von Blutstühlen. In den anderen beiden Gruppen blieb die Darmfunktion weitgehend unverändert.

Die von den Patienten wahrgenommene Lebensqualität spiegelte die Veränderungen in Sexual-, Darm- und Harnfunktionen wider.

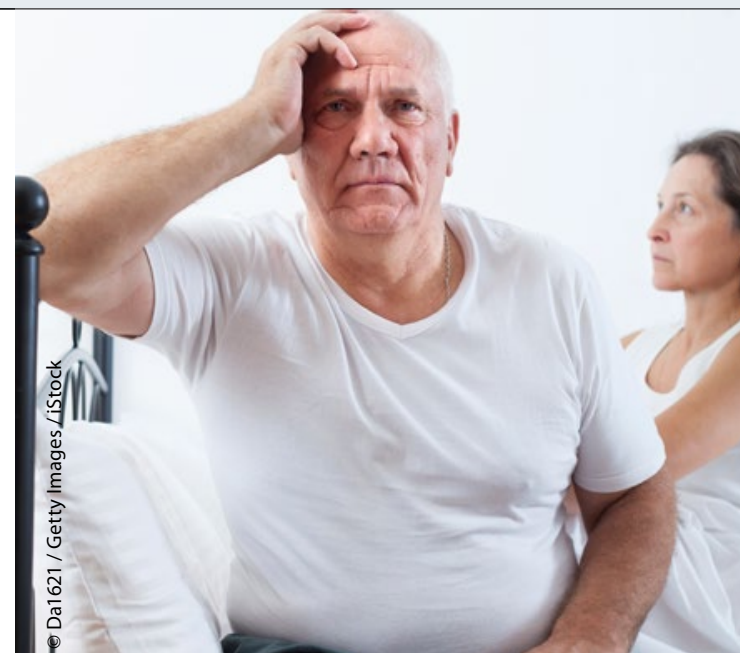

Eine radikale Therapie wirkt sich $u$. a. negativ auf die Sexualfunktion aus.

Fazit: Patienten mit lokal begrenztem Prostatakarzinom erleben, wenn sie sich einer radikalen Therapie unterziehen, Einschränkungen in Sexual-, Harn- und Darmfunktionen, die sich auch auf die Lebensqualität auswirken. Die Nachbeobachtungszeit der Studie sollte, so die Empfehlung der Forscher, auf mindestens 10 Jahre ausgeweitet werden, um die Langzeiteffekte zu beobachten.

Kathrin von Kieseritzky

Donovan JL et al. Patient-Reported Outcomes after Monitoring, Surgery, or Radiotherapy for Prostate Cancer. N Engl J Med. 2016; 375(15): 1425-37.

\section{PCa.: Welche Strategie ist langfristig die beste?}

\section{Beim lokal begrenzten Prostatakrebs ist aktive Beobachtung ebenso möglich wie radikale Prostatektomie oder externe Bestrahlung. Wie sieht das Lang- zeitüberleben unter diesen drei Strategien aus?}

$\mathrm{D}$ erzeit wird kontrovers diskutiert, welches Vorgehen beim lokal begrenzten Prostatakarzinom, das mittels PSA-Test entdeckt wurde, zu bevorzugen ist. Nun gibt es Langzeitergebnisse einer Studie zur Effizienz der drei Behandlungsstrategien „aktives Beobachten“, „radikale Prostatektomie“ und „externe Radiotherapie“. Von 82.429 Männern zwischen 50 und 69 Jahren hatten 2.664 aufgrund eines PSA-Tests die Diagnose eines lokal begrenzten Prostatakarzinoms erhalten. Von ihnen wurden 1.643 randomisiert entweder aktivem Monitoring, einer radikalen Prostatektomie oder einer Radiotherapie zugewiesen. Primä- rer Endpunkt war die Prostatakarzinomspezifische Mortalität nach median zehn Jahren. Sekundäre Endpunkte waren Progressionsrate, Metastasenhäufigkeit und allgemeine Sterberate.

17 Patienten starben am Prostatakarzinom: 8 in der aktiven Beobachtungsgruppe $(1,5$ Todesfälle pro 1.000 Personenjahre), 5 in der Operationsgruppe (0,9/1.000) und 4 in der Radiotherapiegruppe $(0,7 / 1.000)$. Die Unterschiede waren nicht signifikant $(\mathrm{p}=0,48)$. Ebenfalls keine signifikanten Unterschiede ergaben sich in Bezug auf die allgemeine Mortalität (169 Todesfälle insgesamt; $p=0,87$ ). Bei den Patienten der aktiven Beobachtungsgrup- pe traten mehr Metastasen auf (6,3/1.000) als in der Operations- $(2,4 / 1.000)$ und in der Radiotherapiegruppe (3,0/1.000; $\mathrm{p}=0,004)$. Auch schritt die Krankheit in der aktiven Beobachtungsgruppe häufiger voran (22,9 Ereignisse pro 1.000 Personenjahre) als in der Operationsgruppe $(8,9 / 1.000)$ und in der Strahlentherapiegruppe $(9,0 / 1.000 ; \mathrm{p}<0,001)$.

Fazit: Nach median zehn Jahren war die Prostatakarzinom-bezogene Mortalität unabhängig von der gewählten Therapiestrategie niedrig, signifikante Unterschiede gab es zwischen den Gruppen nicht. Nach Operation und Radiotherapie kam es seltener als bei aktivem Monitoring zu Krankheitsprogress und Metastasenbildung. Kathrin von Kieseritzky

Hamdy FC et al. 10-Year Outcomes after Monitoring, Surgery, or Radiotherapy for Localized Prostate Cancer. N Engl J Med. 2016; 375(15): 1415-24. 